\title{
Invalid ECG Waveforms
}

National Cancer Institute

\section{Source}

National Cancer Institute. Invalid ECG Waveforms. NCI Thesaurus. Code C114171.

An electrocardiog raphic recording for which the displayed leads do not represent the individual's true ECG lead information. (CDISC) 